\title{
Prospective study of relations between cortical Lewy bodies, poor eyesight, and hallucinations in Alzhemier's disease
}

\author{
R McShane, K Gedling, M Reading, B McDonald, M M Esiri, T Hope
}

\begin{abstract}
The presence of hallucinations is included in some, but not all, of the sets of clinical diagnostic criteria that have been proposed for dementia associated with cortical Lewy bodies. These criteria were developed from retrospective casenote analyses. This prospective, longitudinal study suggests that, in patients with Alzheimer's disease, cortical Lewy bodies are associated with more persistent and severe hallucinations, independently of any association with severity of cognitive decline. Poor eyesight contributes to the severity but not the persistence of the hallucinations.
\end{abstract}

(F Neurol Neurosurg Psychiatry 1995;59:185-188)

Keywords: Lewy body; dementia; hallucinations

It has been suggested that patients with dementia who have cortical Lewy bodies are prone to develop florid visual hallucinations. ${ }^{1-4} \mathrm{Up}$ to $49 \%$ of patients with Alzheimer's disease, however, have hallucinations at some point in their illness, ${ }^{5}$ and many patients with cortical Lewy bodies also have at least some of the neuropathological changes of Alzheimer's disease. ${ }^{6-9}$ Furthermore, it has been shown that hallucinations are associated with poor eyesight in elderly people $^{10}$ and with more severe cognitive deficits in dementia. ${ }^{11}$ Whether there is any specific connection between Lewy bodies and hallucinations therefore remains unclear.

We report here the results of the first longitudinal study of clinicopathological correlates of cortical Lewy body disease. We have restricted our sample to patients with neuropathologically diagnosed Alzheimer's disease to assess any additional effect of cortical Lewy body pathology on the persistence and severity of hallucinations. We have also examined the effect on hallucinations of two other potential confounding factors-namely, poor eyesight and poor cognitive function.

\section{Methods}

PATIENTS AND CLINICAL ASSESSMENTS

A series of 98 patients who satisfied DSMIII-
$\mathbf{R}$ criteria for dementia were recruited and assessed every four months over a period of up to five years until death. All patients were initially living at home with carers who were able to give a clear account of the patients' behaviour. Their behaviour and mental health were assessed with the present behavioural examination (PBE). ${ }^{12}$ This is a semistructured interview that examines symptoms in the four weeks before the interview. It was given to the main carer by trained interviewers. The cognitive function of patients was assessed with the mini mental state examination (MMSE). ${ }^{13}$

As part of the PBE carers were asked the question "Over the past four weeks, do you think (...patient...) has heard or seen things which are not really there? For example, heard imaginary voices, or seen imaginary things?". The interviewers asked questions of clarification as necessary to decide whether patients had experienced hallucinations. If present, hallucinations were rated on a seven point frequency scale, corresponding to the number of days over the four week period on which the symptom had occurred. Pilot versions of the PBE suggested that distinctions between auditory and visual hallucinations are unreliable in patients with dementia. Therefore, such symptoms were rated under one item. It is often difficult to make the distinction between hallucinations and illusions in patients with dementia. Interviewers were careful to make this distinction, however, and rated hallucinations as present only if the evidence was good. They also recorded brief clinical descriptions of the symptoms being rated. Symptoms which could have been related to temporary physical illness or to changes in medication were rated as "missing".

From these data we have derived two measures for the severity of hallucinations: mean severity is the mean score per interview of the seven point rated item; and long term persistence is the proportion of all interviews at which hallucinations had been rated positively. Poor eyesight was rated as present if the patient's corrected eyesight was ever "too poor to make out images on a TV set or very large print" and the persistence of poor eyesight is the proportion of all interviews at which poor eyesight was present. Raters were 
careful to distinguish between visual agnosias and problems of acuity.

\section{NEUROPATHOLOGY}

Necropsy material was assessed for Alzheimer's disease with methenamine silver and Cross modification of Palmgren silver stains. The number of Lewy bodies was counted in parallel sweeps over the whole of the following four limbic cortical areas stained with haematoxylin and eosin $(\mathrm{H}$ and E) and with antiubiquitin antiserum: $1 \mathrm{~cm}$ strips of insula cortex at the level of the amygdala; the whole of the anterior cingulate gyrus; parahippocampal gyrus at the level of the lateral geniculate body; and entorhinal cortex at the level of the amygdala (from half way along the gyrus to the base of the collateral sulcus). In counting each $\mathrm{H}$ and $\mathrm{E}$ stained section the assessor was blind to Lewy body pathology in the substantia nigra; in each of the other $\mathrm{H}$ and $\mathrm{E}$ stained cortical areas; and in the sections stained with antiubiquitin; Alzheimer's disease pathology on the silver stains; and clinical information. We found it easier to distinguish between small tangles and Lewy bodies in the $\mathrm{H}$ and $\mathrm{E}$ stained than in the material stained with antiubiquitin. Various criteria for the presence of significant numbers of cortical Lewy bodies have been proposed. ${ }^{1415}$ For this analysis we adopted the criterion that at least one Lewy body should be present in at least two of the four $\mathrm{H}$ and $\mathrm{E}$ stained cortical areas, as this was sufficient to eliminate putative false positives in which Lewy bodies were counted as present in cortical areas but were not seen in the substantia nigra.

\section{Results}

NEUROPATHOLOGY

At the time of this study, 86 of the patients had died. Fixed brain material from neocortical and limbic areas was available for 44 patients. The Consortium to Establish a Registry for Alzheimer's Disease (CERAD) neuropathological criteria for probable or definite Alzheimer's disease were satisfied in 41 patients. ${ }^{16}$ Eight of the 41 patients satisfied our criteria for the presence of cortical Lewy bodies.

\section{CLINICAL FINDINGS}

In the sample of 41 patients with Alzheimer's disease, the median age at death was 81 (range 63-97) years and 20 were men. The mean duration until death of reported dementia was $7 \cdot 4$ (SD 3.2) years. Carers were interviewed a median of eight (range 1-15) times over a mean of 2.7 years. The median MMSE score at entry was 13 (range 0-26). No patient had a clinical diagnosis of Parkinson's disease. Thirteen of the 41 patients were institutionalised during the course of the study. Those with hallucinations or cortical Lewy bodies were not more likely to have been institutionalised than those without. There was no difference in the severity or persistence of hallucinations before and after institutionalisation.
Four patients had brief trials of antiparkinsonian medication but this was not associated with any increase in the scores for hallucinations and no patient was taking antiparkinsonian medication at more than one interview. These four were more likely to have cortical Lewy bodies than would be expected by chance (Fisher's exact test; $P=0.02$ ). There was no evidence that treatment with neuroleptic drugs resulted in suppression of the scores for hallucinations. Indeed, among the 22 patients who ever received neuroleptic drugs at any point in the study, there was a non-significant trend for the scores for hallucinations to be greater at interviews at which patients were also recorded as taking neuroleptic drugs than at interviews when they were not $(P=0.09)$. Patients with hallucinations or cortical Lewy bodies were not more likely to have taken neuroleptic drugs.

\section{CLINICOPATHOLOGICAL CORRELATIONS}

Of the 41 subjects with Alzheimer's disease, 13 had hallucinations at some point during the study and five of these had cortical Lewy bodies. Of the 28 patients without hallucinations, three had cortical Lewy bodies. There was a trend, which just failed to reach significance (Fisher's exact test; $P=0.051$ ), for patients with cortical Lewy bodies to be more likely to have had hallucinations at some point during the study than those without. Patients with cortical Lewy bodies were, however, likely to have been much more persistently and severely hallucinated than those without. Among those with cortical Lewy bodies, hallucinations were present on almost half of all interviews (0.44 (SEM 0.15)), whereas hallucinations were present on less than one in $10(0.06(0.03))$ of all interviews in those without cortical Lewy bodies $(\mathrm{P}<$ $0.001)$. Similarly, the mean score for hallucinations on the seven point scale was much greater in those with $(1.3(0.54))$ than in those without $(0.073(0.034))$ cortical Lewy bodies $(P<0.001)$.

We examined data from the whole cohort of 98 patients to establish which factors might be associated with the occurrence of hallucinations. There were no significant associations with sex, age at onset of dementia or death, duration of dementia, or the occurrence of hearing problems. Patients who had hallucinations were significantly more likely to have had poor eyesight $\left(\chi^{2}=6.3, P=0.012\right)$ at some point during the study but there was no significant correlation between the persistence of visual problems and the persistence of hallucinations $(P=0 \cdot 28, r=0 \cdot 17)$. Those who had ever had hallucinations (even if at only one interview) had significantly lower MMSE scores at their last interview (8.5 v $3.5, P=0.005)$. Within the group of 41 patients assessed neuropathologically as having Alzheimer's disease; however, a similar trend for the final MMSE to be lower in those who had ever had hallucinations did not reach significance $(6.8 v 3 \cdot 8, \mathrm{P}=0 \cdot 14)$. There was also a trend for those with Lewy bodies to have lower MMSE scores than 
those without $(6.5 v 3.5, P=0.19)$. The mean (SEM) final MMSE scores for different subgroups were: $3 \cdot 4(2 \cdot 5)$ for those with Lewy bodies and hallucinations; $3 \cdot 3(3 \cdot 3)$ for those with Lewy bodies and without hallucinations; $4 \cdot 0(1 \cdot 8)$ for those with hallucinations but no Lewy bodies; $7 \cdot 2(1 \cdot 6)$ for those without hallucinations or Lewy bodies.

To assess the independence of the possible contributions which cortical Lewy bodies, cognitive decline, and poor eyesight might be making to the severity of hallucinations, we entered them into two analyses of covariance (table). The dependent variables were the two derived measures of the severity of hallucinations as defined earlier. This confirmed that both the mean severity of hallucinations and the long term persistence of hallucinations were significantly and independently associated with the presence of cortical Lewy bodies. Poor vision had a significant effect on the short term severity but not the long term persistence of hallucinations. Cognitive decline did not have a significant independent effect on these measures of the severity of hallucinations. The presence of cortical Lewy bodies accounted independently for $22.3 \%$ of the variance in the short term severity of hallucinations and $26.2 \%$ of the variance in the long term persistence of hallucinations.

There was a significant two way interaction between the presence of cortical Lewy bodies and poor eyesight in the severity of the hallucinations, suggesting the possibility that the effect of Lewy bodies is different in those with and those without visual problems. In patients who had visual problems, those with Lewy bodies had significantly $(P=0.013)$ more severe hallucinations (mean severity: $2.02(0.78))$ than those without $(0.053$ $(0.04))$. In those without visual problems, the trend for those with Lewy bodies to have more severe hallucinations than those without did not reach significance (mean severity in patients with and without Lewy bodies 0.33 (0.33) $v 0.09(0.043): \mathrm{P}=0.14 ; 95 \%$ confidence interval $(95 \% \mathrm{CI})-0.57$ to 0.09$)$.

The raw data showed that three of the five patients with both cortical Lewy bodies and hallucinations had hallucinations on

Covariance of parameters with severity and persistence of hallucinations

\begin{tabular}{|c|c|c|c|c|c|c|}
\hline & \multicolumn{3}{|c|}{$\begin{array}{l}\text { Short term severity of } \\
\text { hallucinations }\end{array}$} & \multicolumn{3}{|c|}{$\begin{array}{l}\text { Long term persistence of } \\
\text { hallucinations }\end{array}$} \\
\hline & $\begin{array}{l}\text { Sum of } \\
\text { squares }\end{array}$ & (F) & $P$ value & $\begin{array}{l}\text { Sum of } \\
\text { squares }\end{array}$ & (F) & Pvalue \\
\hline $\begin{array}{l}\text { Main effects: } \\
\text { Cortical Lewy body } \\
\text { Visual problems }\end{array}$ & $\begin{array}{l}6 \cdot 8 \\
3 \cdot 6\end{array}$ & $\begin{array}{r}(17 \cdot 2) \\
(9 \cdot 1)\end{array}$ & $\begin{array}{r}<0.001 \\
0.005\end{array}$ & $\begin{array}{l}0.74 \\
0.036\end{array}$ & $\begin{array}{l}(14 \cdot 4) \\
(0 \cdot 70)\end{array}$ & $\begin{array}{l}0.001 \\
0.41\end{array}$ \\
\hline $\begin{array}{l}\text { Covariates: } \\
\text { Last MMSE score }\end{array}$ & 0.003 & $(0.006)$ & 0.94 & 0.010 & $(0 \cdot 19)$ & 0.67 \\
\hline $\begin{array}{l}\text { Two-way interaction: } \\
\text { Lewy body and visual } \\
\text { problems }\end{array}$ & $4 \cdot 1$ & $(10 \cdot 5)$ & 0.003 & 0.062 & $(1 \cdot 13)$ & $0 \cdot 30$ \\
\hline Total & $30 \cdot 4$ & & & 2.85 & & \\
\hline
\end{tabular}

more than five of the previous 28 days at two consecutive interviews (spaced four months apart), whereas this did not apply to any of the 33 patients without cortical Lewy bodies.

\section{Discussion}

In this study of 41 prospectively assessed patients with pathologically confirmed Alzheimer's disease, eight of 41 (19.5\%) also had cortical Lewy bodies. Our findings show that those with cortical Lewy bodies have more persistent and more severe hallucinations than those without. The trend for cortical Lewy bodies to be present, however, in those who have ever had hallucinations was not quite significant $(P=0.051)$. At least one other study of patients presenting to a psychiatric service has failed to show that cortical Lewy bodies are more common in those who have ever had hallucinations. ${ }^{17}$ The apparently conflicting results from different studies may be because patients with Alzheimer's disease but without cortical Lewy bodies sometimes experience occasional brief periods of hallucinosis. The value of longitudinal data is that these brief episodes can be identified as such.

Our findings emphasise the important, and occasionally treatable, contribution which poor eyesight makes to the severity of hallucinations in patients with dementia. The definition of poor eyesight was based on a simple functional rating rather than ophthalmological assessment. It is possible that, despite the careful attempts of interviewers to establish the nature of the phenomena, behaviour which was actually due to patients' poor vision was attributed to hallucinations; however, if this were occurring one would expect the two symptoms to be reported simultaneously because within a one month period, poor eyesight would be apparent on days when the subject was not hallucinating as well as on days when hallucinations occurred. The failure to find a correlation between the persistence over time of the two symptoms suggests that this potentially confounding factor was not an important source of error.

The finding of an interaction between Lewy bodies and visual problems would seem to imply that Lewy bodies were only significantly associated with the severity of hallucinations in those with visual problems. There were, however, only three patients who were Lewy body positive in the group without visual problems and as indicated, the $95 \%$ CIs approached 0 . We are therefore reluctant to attach much relevance to this finding until it is replicated.

Persistent hallucinations should be retained as one of the diagnostic criteria for dementia associated with cortical Lewy body disease. Larger prospective longitudinal studies are required to test whether persistent hallucinations can usefully be further defined in this context as "hallucinations present on more than five days per month over a period of at least four months". 
R H M and M R were funded by the E P Abrahams Research Fund. K G is funded by the Medical Research Council. We are grateful to Dr P Griffiths for statistical advice. We also thank our referees and Dr R Jacoby for their comments on thank our refer
earlier drafts.

1 McKeith IG, Fairbairn AF, Perry RH, Thompson P. The clinical diagnosis and misdiagnosis of senile dementia of Lewy body type (SDLT). Br F Psychiatry 1994;165: 324-32.

2 Crystal HA, Dickson DW, Lizardi JE, Davies P, Wolfson LI. Antemortem diagnosis of diffuse Lewy body disease. Neurology 1990;40:1523-8.

3 Byrne EJ, Lennox GG, Godwin-Austen RB, et al. Dementia associated with cortical Lewy bodies: proposed clinical diagnostic criteria. Dementia 1991;2. 283-4.

4 Hansen L, Salmon D, Galasko D, et al. The Lewy body variant of Alzheimer's disease. Neurology 1990;40:1-8.

5 Wragg RE, Jeste DV. Overview of depression and psychosis in Alzheimer's disease. Am $\mathcal{J}$ Psychiatry 1989;146:577-87.

6 Perry RH, Irving D, Blessed G, Fairbairn A, Perry EK. Senile dementia of Lewy body type. A clinically and neuropathologically distinct form of Lewy body dementia in the elderly. F Neurol Sci 1990;95:119-39.

7 Ince P, Irving D, MacArthur F, Perry RH. Quantitative neuropathological study of Alzheimer-type pathology in the hippocampus: comparison of senile dementia of Alzheimer type, senile dementia of Lewy body type, Parkinson's disease and non-demented elderly control patients. F Neurol Sci 1991;106:142-52.

8 Dickson DW, Schmidt ML, Lee VM, Zhao ML, Yen SH,
Trojanowski JQ. Immunoreactivity profile of hippocampal CA2/3 neurites in diffuse Lewy body disease. Acta Neuropathol (Berl) 1994;87:269-76.

9 Lippa CF, Smith TW, Swearer JM. Alzheimer's disease and Lewy body disease: a comparative clinicopathologiand Lewy body disease: a comparative

10 Teunisse JR, Cruysberg JRM, Verbeek A, Zitman G. The Charles Bonnet syndrome: a large prospective study in The Netherlands. Br $\mathcal{F}$ Psychiatry 1995;166:254-7.

11 Merriam AE, Aronson MK, Gaston P, et al. The psychiatric symptoms of Alzheimer's disease. $¥ \mathrm{Am}$ Geriatr Soc 1988;36:7-12.

12 Hope T, Fairburn CG. The Present Behavioural Examination (PBE): the development of an interview to measure current behavioural abnormalities. Psychol Med 1992;22:223-30.

13 Folstein MF, Folstein SE, McHugh PR. "Mini-mental state": a practical method for grading the cognitive state of patients for the clinician. $\mathcal{F}$ Psychiatr Res 1975;12: of patients

14 Kosaka K. Diffuse Lewy body disease in Japan. $f$ Neurol 1990;237:197-204.

15 Lennox G, Lowe J, Landon M, Byrne EJ, Mayer RJ, Godwin-Austen RB. Diffuse Lewy body disease: correlative neuropathology using anti-ubiquitin immunocytochemistry. $f$ Neurol Neurosurg Psychiatry 1989;52: 1236-47.

16 Mirra SS, Hart MN, Terry RD. Making the diagnosis of Alzheimer's disease. A primer for practicing pathologists. Arch Pathol Lab Med 1993;117:132-44

17 Forstl H, Burns A, Luthert P, Cairns N, Levy R. The Lewy-body variant of Alzheimer's disease. Clinical and pathological findings. Brf Psychiatry 1993;162:385-92.

\section{A note on Claude Bernard-Horner's syndrome}

Claude Bernard (1813-78), born in St Julien in Rhone, reported in 1852 his experiments on cutting the rabbit's cervical sympathetic nerve. He observed that this caused constriction of the ipsilateral pupil, but also flushing and a rise in temperature of the ear. From this he deduced for the first time that sympathetic nerves (vasomotor) controlled flow in blood vessels. ${ }^{1}$

Horner described the triad of ptosis, miosis, and enophthalmos in 1869 . Though known by his name, earlier, but sometimes incomplete, accounts exist in the writings of Pourfour du Petit. ${ }^{2}$ Weir Mitchell ${ }^{3}$ however, gave the first full account five years before Horner, describing a 24 year old soldier with a gunshot wound of the right side of his neck:

"The pupil of the right eye is very small ... slight but very distinct ptosis . . . The ball of the right eye looks smaller than that on the left ... The conjunctiva is somewhat redder... and the pupil somewhat deformed, oval rather than round ... his face became distinctly flushed on the right side only when walking in warm weather ... a case of injury of the sympathetic nerve, probably the only one recorded."

The soldier's signs recovered in five months and he returned to military duties.

Thirty years earlier Edward Selleck Hare, House Surgeon to Stafford County General Infirmary, had described the physical signs in a letter ${ }^{4}$ to the Medical Gazette on 11 September 1838. His patient was a Thomas Willetts aged 40 with:

"pain, tingling and numbness along the course of the ulnar nerve of the left arm, ... and a small tumour in the inferior triangular space on the left side of the neck ... the pupil of the left eye became contracted; and the levator palpebrae ceased to perform its office."... He died within 10 weeks. Postmortem showed extensive infiltration of the brachial plexus, jugular and carotid vessels, anterior mediastinum, vagus and "the sympathetic, with its lower cervical ganglion." Hare could not relate the ocular signs to the structural disease, "they must be regarded as an instance of that remote sympathy which is found to exist between distant parts of the same individual ...."

Sadly, Hare died on 28 September 1838 aged 26 of typhoid fever.

Horner's paper ${ }^{5}$ was translated by Fulton ${ }^{6}$ :

"Anna Brändli, aged 40, a healthy looking peasant

woman... six weeks after her last confinement noticed a slight drooping of her right upper eyelid, which increased very gradually... The upper lid covers the right cornea to the upper edge of the pupil; the lid is not loose or wrinkled but somewhat sunken into the orbit and is still capable of movement; it is neither injected nor swollen. The upper convex furrows of the forehead indicate that the frontalis muscle is working as a substitute [for the levator palpebrae superioris]

The pupil of the right eye is considerably more constricted than that of the left, but reacts to light; the globe has sunk inward very slightly ... During the clinical discussion... the right side of her face became red and warm; while the left side remained pale and cool. The right side seemed turgid and rounded, the left more sunken and angular; the one perfectly dry, the other moist. The boundary of the redness and warmth was exactly in the midline. The patient thereupon told us that the right side had never perspired . . I Immediately after application, the thermometer on the right recorded $35 \mathrm{C}$, that on the left, 30C, ..."

Horner measured temperature behind the ear and in the axilla and groin.

"After application of atropine into each conjunctival sac the right pupil enlarged slowly and irregularly; after twenty minutes it had not reached the size of the left... When 24 hours after atropine, equal quantities of calabar ${ }^{\star}$ were put into the conjunctival sac of each eye, one noticed after ten minutes a marked constriction on the right; while on the left the action of atropine still continued ..."

Considering the cause of each sign in turn, Horner concluded:

"the vasomotor disturbance involves not only the trigeminal area, but also the fibres of the cervical sympathetic; this experiment with belladonna and calabar speaks for the dual control of the movements of the iris in man ... we are dealing with right dilator paralysis ... Ptosis ... a paralysis of the musculus palpebrae superioris supplied by the sympathetic nerve ( $H$. Müller, Harling), and the appearance of the upper lid as part and parcel of the whole symptom-complex."

Johann Friedrich Horner (1831-86) was born in Zurich. His medical studies were profoundly influenced by the physiologist Karl Ludwig. After graduating in 1854 he visited Munich and Vienna and continued on page 191

* Calabar is the extract from the seed of a woody vine Physostigma venenosum containing physostigmine, which was used by Argyll Robertson in 1863 for examining the pupils. 